\title{
Preventing cytokine storm syndrome in COVID-19 using $\alpha-1$ adrenergic receptor antagonists
}

\author{
Maximilian F. Konig, ${ }^{1,2,3,4,5}$ Mike Powell, ${ }^{6}$ Verena Staedtke, ${ }^{7}$ Ren-Yuan Bai, ${ }^{8}$ David L. Thomas, ${ }^{9}$ Nicole Fischer, ${ }^{5}$ Sakibul Huq, ${ }^{8}$ \\ Adham M. Khalafallah, ${ }^{8}$ Allison Koenecke, ${ }^{10}$ Ruoxuan Xiong, ${ }^{11}$ Brett Mensh, ${ }^{12}$ Nickolas Papadopoulos, ${ }^{1,2,3}$ Kenneth W. Kinzler, ${ }^{1,2,3}$ \\ Bert Vogelstein,, ${ }^{1,2,3}$ Joshua T. Vogelstein, ${ }^{6}$ Susan Athey, ${ }^{13}$ Shibin Zhou, ${ }^{1,2,3}$ and Chetan Bettegowda ${ }^{1,2,3,8}$
}

'Ludwig Center, ${ }^{2}$ Lustgarten Laboratory, and ${ }^{3}$ Howard Hughes Medical Institute, Johns Hopkins Kimmel Cancer Center, Baltimore, Maryland, USA. ${ }^{4}$ Division of Rheumatology, ${ }^{5}$ ohns Hopkins University (JHU) School of Medicine, Baltimore, Maryland, USA. ${ }^{6}$ Department of Biomedical Engineering, Institute of Computational Medicine, JHU, Baltimore, Maryland, USA. 'Department of Neurology, ${ }^{7}$ Department of Neurosurgery, and ${ }^{9}$ Division of Infectious Diseases, JHU School of Medicine, Baltimore, Maryland, USA. ${ }^{10}$ Institute for Computational and Mathematical Engineering and ${ }^{11}$ Management Science and Engineering, Stanford University, Stanford, California, USA. ${ }^{2}$ Janelia Research Campus, Howard Hughes Medical Institute, Ashburn, Virginia, USA. ${ }^{3}$ Stanford Graduate School of Business, Stanford University, Stanford, California, USA.

D ysregulated host immune responses drive mortality in pneumonia and acute respiratory distress syndrome (ARDS) caused by a wide range of infections. In coronavirus disease 2019 (COVID-19), severe acute respiratory syndrome coronavirus 2 (SARS-CoV-2) elicits an exuberant local or systemic immune response (hyperinflammation) in the lung and other sites of viral replication, compromising organ function and leading to high morbidity and mortality (1-4).

\section{Cytokine storm syndrome in COVID-19}

Physiologic immune responses are coordinated and self-resolving, whereas uncontrolled immune activation in some patients with infection, autoimmune rheumatic disease, or chimeric antigen receptor- $\mathrm{T}$ cell (CAR-T cell) therapy results in syndromes of hyperinflammation. These syndromes are characterized by the overproduction of cytokines and other secreted proinflammatory molecules. Emerging evidence suggests that a subset of patients with COVID-19 develops a cytokine storm syn- drome (CSS) that is associated with elevation of proinflammatory cytokines, including IL-6, IL-2R, IL-8, TNF- $\alpha$, and G-CSF $(2,4-8)$, similar to the excessive cytokine production by lung-infiltrating monocytes/ macrophages and pneumocytes observed in SARS-CoV-1 and Middle East respiratory syndrome-CoV (MERS-CoV) infection (9). Alveolar inflammation and diffuse alveolar damage impair the infected lungs' ability to participate in gas exchange, culminating in ARDS and necessitating mechanical ventilation (10). ARDS is the main driver of mortality of COVID-19, so preventing the hyperinflammation is critical for avoiding this progression.

\section{Treating cytokine storm in COVID-19}

One potential therapeutic target is the IL-6 signaling pathway. IL-6 levels diverge profoundly between survivors and nonsurvivors in the third week after symptom onset and predict COVID-19 severity and in-hospital mortality $(1,8,11)$. Tocilizumab and sarilumab, monoclonal antibodies targeting the IL-6 receptor, and siltuximab, a
Conflict of interest: The JHU filed a patent application on the use of various drugs to prevent cytokine release syndromes, on which VS, RYB, NP, BV, KWK, and SZ are listed as inventors. JHU will not assert patent rights from this filing for treatment related to COVID-19. MFK received personal fees from Bristol-Myers Squibb and Celltrion. BV, KWK, and NP are founders of and hold equity in Thrive Earlier Detection. KWK and NP are consultants to and are on the Board of Directors of Thrive Earlier Detection. BV, KWK, NP, and SZ are founders of, hold equity in, and serve as consultants to Personal Genome Diagnostics. SZ holds equity in Thrive Earlier Detection and has a research agreement with BioMed Valley Discoveries Inc. KWK and BV are consultants to Sysmex, Eisai, and CAGE Pharma and hold equity in CACE Pharma. NP is an advisor to and holds equity in Cage Pharma. BV is also a consultant to Nexus. KWK, BV, SZ, and NP are consultants to and hold equity in NeoPhore. CB is a consultant to Depuy-Synthes and Bionaut Pharmaceuticals. CB, BV, KWK, and NP are also inventors on technologies unrelated or indirectly related to the work described in this article. Licenses to these technologies are or will be associated with equity or royalty payments to the inventors, as well as to JHU. The terms of all these arrangements are being managed by JHU in accordance with its conflict of interest policies. SA is an advisor and holds an equity stake in two private companies, Prealize (Palo Alto, Callifornia, USA) and Consulta (Brazil). Prealize is a health care analytics company, and Consulta operates a chain of low-cost medical clinics in Brazil.

Copyright: () 2020, American Society for Clinical Investigation.

Reference information: J Clin Invest. 2020;130(7):3345-3347. https://doi.org/10.1172/JCl139642. chimeric antibody targeting IL-6, are currently being investigated for the treatment of patients with COVID-19-CSS (12-23). Pending data from randomized controlled trials, retrospective data from 21 patients with severe or critical COVID-19 treated with tocilizumab suggest that inhibiting the IL-6 signaling axis may reduce patient morbidity and the need for mechanical ventilation (24) but may fail to treat very advanced disease (25). However, given the cost, immunosuppression, and potential adverse reactions of tocilizumab, this strategy will likely be restricted to select patients in developed countries.

\section{Preventing cytokine storm by targeting the catecholamine- cytokine axis}

We have shown that CSS, observed with bacterial infections, CAR-T cells, and other $\mathrm{T}$ cell-activating therapies, is accompanied by a surge in catecholamines (26). Catecholamines enhance inflammatory injury by augmenting the production of IL- 6 and other cytokines through a self-amplifying feed-forward loop in immune cells that requires $\alpha-1$ adrenergic receptor $\left(\alpha_{1}\right.$-AR) signaling (26). Prophylactic inhibition of catecholamine synthesis with metyrosine, a tyrosine hydroxylase antagonist, reduced levels of catecholamines and cytokine responses and resulted in markedly increased survival following various inflammatory stimuli in mice. Similar protection against a hyperinflammatory stimulus was observed with the well-tolerated $\alpha_{1}$-AR antagonist, prazosin (but not $\beta$-AR antagonists), demonstrating that this class of drugs can also prevent cytokine storm (26).

Preliminary results from a recent retrospective clinical study revealed that, for hospitalized patients diagnosed with pneumonia 
or acute respiratory distress, the likelihood of requiring mechanical ventilation and dying was significantly lower if patients were taking $\alpha_{1}$-AR antagonists during the year preceding hospitalization (27)

\section{Need for clinical trials}

These findings offer a rationale for studying $\alpha_{1}$-AR antagonists to prevent CSS and its dire consequences in people who are at risk for developing severe COVID-19. This population includes people who are recently infected with SARS-CoV-2 and people who are not yet infected but are at high risk for exposure. Prazosin is inexpensive and safe, as documented by long-term treatment of millions of patients with benign prostatic hyperplasia, hypertension, and other conditions. However, all drugs can have unanticipated side effects in different clinical contexts, and the incompletely understood relationship between hypertension and COVID-19 suggests caution in using any agent that affects blood pressure (28). Prospective clinical trials in high-risk patients are needed to assess $\alpha_{1}$-AR antagonist utility in preventing - not treating - COVID-19-CSS. We emphasize that the extensive experience with using prazosin for other indications should prioritize not obviate - rigorous, controlled clinical research rather than indiscriminate off-label use in patients exposed to or infected with SARS-CoV-2. Such trials could be expeditiously implemented in areas suffering from high infection rates that are overwhelming hospital capacity. To that end, we are actively pursuing clinical trials at multiple institutions and will make our protocols available on http://clinicaltrials.gov/ when approved by the Johns Hopkins Internal Review Board. The potential therapeutic benefit of $\alpha_{1}$-AR antagonism may extend beyond COVID-19. The potential utility of prazosin prophylaxis and early abortive therapy in the prevention of morbidity and mortality in ARDS, pneumonia, CAR-T cell therapy, and autoimmune rheumatic disease deserves dedicated study.

\section{Acknowledgments}

MFK was supported by the National Institute of Arthritis and Musculoskeletal and Skin Diseases of the NIH under award no. T32AR048522. AK was supported by the National Science Foundation Graduate Research Fellowship under grant DGE-
1656518. Any opinion, findings, and conclusions or recommendations expressed in this material are those of the authors and do not necessarily reflect the views of the National Science Foundation. JV and SA were partially supported by funding from Microsoft Research. CB was supported by the Burroughs Wellcome Career Award for Medical Scientists, National Cancer Institute grant R37CA230400, National Institute of Neurological Disorders and Stroke grant R21NS113016, and Department of Defense grant W81XWH-16-0078. VS was supported by the National Cancer Institute grant K08CA230179 and U01CA247576. VS is also a recipient of The Sontag Foundation Distinguished Scientist Award. RYB was supported by the National Cancer Institute grant U01CA247576 and the Department of Defense grant W81XWH1810236. This work was further supported by the Virginia and D.K. Ludwig Fund for Cancer Research, the Lustgarten Foundation for Pancreatic Cancer Research, and the BKI Cancer Genetics and Genomics Research Program.

Address correspondence to: Chetan Bettegowda, Johns Hopkins University School of Medicine, 600 North Wolfe Street 21287, Phipps 118, Baltimore, Maryland 21287, USA. Phone: 410.955.8620; Email: cbetteg1@jhmi.edu. Or to: Shibin Zhou, Johns Hopkins University School of Medicine, 1650 Orleans Street, Room 590, Baltimore, Maryland 21231, USA. Phone: 410.955.8878; Email sbzhou@jhmi.edu. Or to: Susan Athey, Stanford Graduate School of Business, 655 Knight Way, Stanford, California 94305, USA. Phone: 650.678.9384; Email: athey@stanford. edu. Or to: Joshua T. Vogelstein, Johns Hopkins University, Center for Imaging Science, Clark Hall, Room 314D, 3400 North Charles Street, Baltimore, Maryland 21218, USA.Email: jovo@jhu.edu.

1. Zhou F, et al. Clinical course and risk factors for mortality of adult inpatients with COVID-19 in Wuhan, China: a retrospective cohort study. Lancet. 2020;395(10229):1054-1062.

2. Ruan Q, Yang K, Wang W, Jiang L, Song J. Clinical predictors of mortality due to COVID19 based on an analysis of data of 150 patients from Wuhan, China [published online March 3, 2020]. Intensive Care Med. https://doi. org/10.1007/s00134-020-06028-z.

3. Qin C, et al. Dysregulation of immune response in patients with COVID-19 in Wuhan, China [published online March 12, 2020]. Clin Infect Dis. https://doi.org/10.1093/cid/ciaa248.

4. Huang C, et al. Clinical features of patients infected with 2019 novel coronavirus in Wuhan, China. Lancet. 2020;395(10223):497-506.

5. Mehta P, et al. COVID-19: consider cytokine storm syndromes and immunosuppression. Lancet. 2020;395(10229):1033-1034

6. McGonagle D, Sharif K, O'Regan A, Bridgewood C. The role of cytokines including interleukin-6 in COVID-19 induced pneumonia and macrophage activation syndrome-like disease [published online April 3, 2020]. Autoimmun Rev. https://doi.org/10.1016/j.autrev.2020.102537.

7. Pedersen SF, Ho Y-C. SARS-CoV-2: a storm is raging. J Clin Invest. 2020;130(5):2202-2205.

8. Chen $\mathrm{G}$, et al. Clinical and immunologic features of severe and moderate coronavirus disease 2019. JClin Invest. 2020;130(5):2620-2629.

9. Channappanavar R, Perlman S. Pathogenic human coronavirus infections: causes and consequences of cytokine storm and immunopathology. Semin Immunopathol. 2017;39(5):529-539.

10. Xu Z, et al. Pathological findings of COVID-19 associated with acute respiratory distress syndrome. Lancet Respir Med. 2020;8(4):420-422.

11. Liu T, et al. The potential role of IL- 6 in monitoring severe case of coronavirus disease 2019. Posted on medRxiv March 10, 2020. https://doi. org/10.1101/2020.03.01.20029769.

12. Tocilizumab in COVID-19 pneumonia (TOCIVID-19). https://clinicaltrials.gov. NCT04317092. Accessed May 1, 2020.

13. Clinical trial of combined use of hydroxychloroquine, azithromycin, and tocilizumab for the treatment of COVID-19. https://clinicaltrials. gov. NCT04332094. Accessed May 1, 2020.

14. Tocilizumab to prevent clinical decompensation in hospitalized, non-critically ill patients with COVID-19 pneumonitis. https://clinicaltrials.gov. NCT04331795. Accessed May 1, 2020.

15. A study to evaluate the safety and efficacy of tocilizumab in patients with severe COVID19 Pneumonia. https://clinicaltrials.gov. NCT04320615. Accessed May 1, 2020.

16. Tocilizumab vs CRRT in management of cytokine release syndrome (CRS) in COVID19. https://clinicaltrials.gov. NCT04306705. Accessed May 1, 2020.

17. Favipiravir combined with tocilizumab in the treatment of corona virus disease 2019. https://clinicaltrials.gov. NCT04310228. Accessed May 1, 2020.

18. Treatment of COVID-19 patients with antiinterleukin drugs. https://clinicaltrials.gov. NCT04330638. Accessed May 1, 2020.

19. Anti-il6 treatment of serious COVID-19 disease with threatening respiratory failure. https:// clinicaltrials.gov. NCT04322773. Accessed May 1, 2020.

20. Evaluation of the efficacy and safety of sarilumab in hospitalized patients with COVID19. https://clinicaltrials.gov. NCT04315298. Accessed May 1, 2020.

21. Sarilumab COVID-19. https://clinicaltrials.gov. NCT04327388. Accessed May 1, 2020.

22. Cohort multiple randomized controlled trials 
open-label of immune modulatory drugs and other treatments in COVID-19 patients. https://clinicaltrials.gov. NCT0 4324073. Accessed May 1, 2020.

23. An observational case-control study of the use of siltuximab in ARDS patients diagnosed with COVID-19 infection. https://clinicaltrials.gov. NCT04322188. Accessed May 1, 2020.

24. Xu X, et al. Effective treatment of severe
COVID-19 patients with tocilizumab. Posted on ChinaXiv March 5, 2020. http://www.chinaxiv. org/abs/202003.00026.

25. Luo $\mathrm{P}$, et al. Tocilizumab treatment in COVID19: a single center experience [published online April 6, 2020]. J Med Virol. https://doi. org/10.1002/jmv.25801.

26. Staedtke V, et al. Disruption of a self-amplifying catecholamine loop reduces cytokine release syndrome. Nature. 2018;564(7735):273-277.

27. Vogelstein JT, et al. Alpha-1 adrenergic receptor antagonists prevent acute respiratory distress syndrome and death: implications for Coronavirus disease 2019. Posted on arXiv April 21, 2020. https://arxiv.org/abs/2004.10117.

28. Zheng YY, Ma YT, Zhang JY, Xie X. COVID-19 and the cardiovascular system. Nat Rev Cardiol. 2020;17(5):259-260. 